\title{
Prescribed Performance Control for Two-axis Optronic Stabilized Platform
}

\author{
WANG Yexing ${ }^{1}$, LUO Changxin ${ }^{1}$, ZHANG Tao ${ }^{1}$, LIU Yaning ${ }^{1}$ and XIAO Chuhan ${ }^{1}$ \\ ${ }^{1}$ Air and Missile Defense College, Air Force Engineering University, 710051 Xi'an, China
}

\begin{abstract}
Aiming at improving the tracking and stabilizing performance of two-axis optronic stabilized platform with Stribeck friction and uncertain velocity disturbance, a prescribed performance control strategy with unknown initial errors is designed. By designing a new performance function, the limit of traditional prescribed control that the initial error has to be known accurately is broken through. The strategy possesses strong robustness against unknown disturbance, and the state error is restrained to a predefined arbitrary small residual. It is guaranteed that the closedloop system is uniformly ultimately bounded. The simulation results demonstrate the effectiveness of proposed strategy.
\end{abstract}

\section{Introduction}

Optronic stabilized platform(OSP) is a kind of highprecision servo tracking system, which is usually mounted on a mobile carrier for stable tracking of moving targets. Optical tracking system is widely used in robot self-balance system[1], unmanned aerial vehicle[2], airborne aiming system [3] and non-strapdown seeker servo system[4].The control object of the optical tracking system is the optical tracking platform. The main factors that affect the tracking performance are the carrier speed interference and the friction torque between the shaft systems[5][6].Two difficulties when dealing with the improvement of the system performance. One is the interference suppression in order to ensure tracking stability and the other is the system response and accuracy.How to effectively suppress interference in order to ensure the stability of tracking, and how to ensure that the system quickly and accurately track the target are two important aspects to improve system performance.

To realize the system performance with fast dynamic response and high-precision control of OSP, researches have tried varieties of approaches. For classical control structure, the proportional-integral-derivative control has been used to many systems [7], however, the robustness can't be guaranteed. To enhance the robustness of the system, $H_{\propto}$ control is introduced to eliminate the sensitivity for disturbance, however, the robustness conflicts with its performance sharply[8]. Furthermore, sliding mode control is also used to deal with the nonlinearity of OSP systems [9], it enables all system states with arbitrary values to converge to user-specified surface, while its chattering problem is an unavoidable drawback. Observer-based control is also used to eliminate disturbance and gets better performance [10].
Disturbance observer achieves high-precision estimation of interference, while the parameters have to be adjusted intricately. In reference [11], the adaptive RBFNN was designed to estimate and compensate for disturbances to improve the control performance of OSP. However, its control performance was easily affected by the selected upper bound of residual approximation error, and the problem of calculating delay have not been solved yet.

A common characteristic of the aforementioned researches is that the transient and steady performance cannot be prescribed, methods mentioned can only guarantee the convergence of error to a residual set. In this case, a prescribed performance control(PPC) is proposed in[12], based on PPC, it's easy to design a prescribed performance bound to acquire desired transient and steady performance[13]. So far, there has not been application of PPC to OSP.

In this paper, PPC is utilized to improve the performance of OSP in the presence of unknown model perturbation and external disturbance. A new performance function is designed to break through the limitation of knowing the initial errors accurately. The designed scheme does not reside to the information of disturbance, which simplify the controller design and increase the practicality of the algorithm.

The paper is organised as follows:

In Section 2, the OSP model and preliminaries are presented. In Section 3, prescribed performance controller is designed along with the stability analysis. In Section 4, simulations are carried out to demonstrate the effectiveness. 


\section{Problem formulation}

\subsection{Constitution of two-axis optronic stabilized platform}

Fig.1 shows the schematic diagram of two-axis stabilized platform. We can see that stabilized platform consists of two gimbals, which are pitch gimbal and yaw gimbal. From Fig. 1 we can see the relationships between two gimbals: gyroscopes measuring the angular rate of pitch and yaw gimbal, angel sensors measuring the angle of pitch and yaw gimbal, moment sensors measuring the moment of pitch and yaw motor.

The system is driven by two servo motors, the sensor such as imaging sensor is placed in the inner frame.

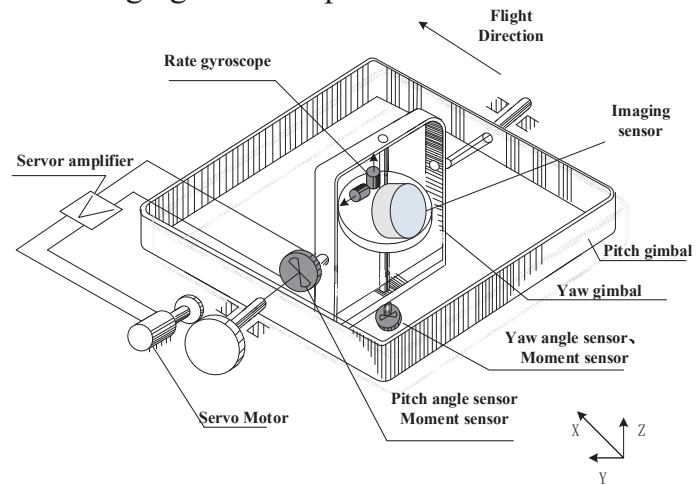

Figure 1. Schematic diagram of the two-axis optronic stabilized platform

\subsection{Dynamic model of the plant}

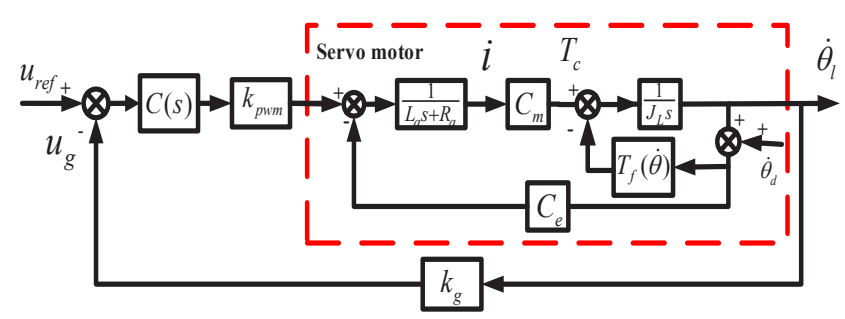

Figure 2. Stabilized-loop block diagram

Fig.2 shows the pitch control block of stabilized platform. Where the block within the red imaginary line stands for the servo motor; $C(s)$ is the controller of stabilized loop; $k_{P W M}$ is the power amplifier coefficient; $T_{l}(\dot{\theta})$ is the moment of friction; $\dot{\theta}_{l}$ is the angular rate of stabilized platform in the inertial space; $\dot{\theta}_{d}$ is the perturbation of angular rate; $T_{f}(\dot{\theta})$ is the friction moment; $k_{g}$ is the simplified transfer function of rate gyroscope, cascade coefficient $1 / k_{g}$ makes it a unit feedback system; $T_{c}$ is the moment output of servo motor.

Combined with the dynamic equation of stable platform and the dynamic equation of motor, mathematical model is acquired as follows [14]:

$$
\left\{\begin{array}{l}
\dot{x}_{1}=\frac{x_{2}}{J_{L}}-\frac{F_{f}\left(x_{1}+\dot{\theta}_{d}\right)}{J_{L}} \\
\dot{x}_{2}=-\frac{C_{m} C_{e}}{L_{a}} x_{1}-\frac{R_{a}}{L_{a}} x_{2}+\frac{C_{m} k_{P W M}}{L_{a}} u-\frac{C_{e} C_{m} \dot{\theta}_{d}}{L_{a}}
\end{array}\right.
$$

Where $x_{1}, x_{2}$ are state variables, they represent $\dot{\theta}_{l}$ and $T_{c}$ respectively. $u$ is the input of controller.

The friction moment is modified as Stribeck friction model as Fig.3[15].

$$
F_{f}(v)=\left[F_{c}+\left(F_{s}-F_{c}\right) e^{-\left(\frac{v}{v_{s}}\right)^{2}}\right] \operatorname{sgn}(v)+B_{v} v(2)
$$

Where $F_{c}, F_{s}$ are coulomb friction and static friction force respectively.

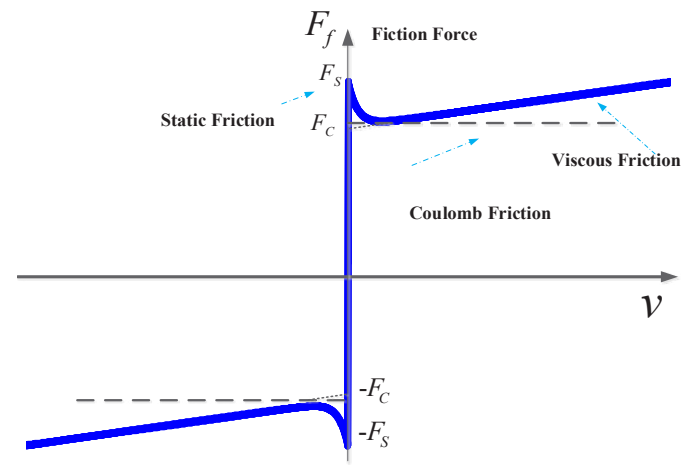

Figure 3. Friction force with Stribeck effect

\subsection{Control problems for the stabilized platform}

There are some troublesome characteristics in the stabilized platform:

1) It is hard to establish accurate mathematical model of the stabilized platform due to its coefficient uncertainty and perturbation;

2) The strong nonlinearity of friction model at velocity zero point may cause dead-area phenomenon of velocity tracking;

3) When there exists unknown external velocity disturbances and internal friction disturbances, it is hard to guarantee the transient and steady state performance.

\section{Controller design}

\subsection{Prescribed performance}

In this subsection, some preliminaries of prescribed performance are summarized. And a new performance function is designed.

Definition 1. [16] A smooth function

$\hbar(t): \square_{+} \rightarrow \square{ }_{+}$will be called a performance function if it satisfies:

(1) $\hbar(t)$ is positive and decreasing;

(2) $\lim _{t \rightarrow+\infty} \hbar(t)=\hbar_{\propto}>0$. 
According to Definition 1, a novel performance function is formulated as:

$$
\hbar_{i}(t)=\operatorname{csch}\left(\kappa_{i} t+\theta_{i}\right)+\hbar_{i \propto}
$$

With $\kappa_{i}, \theta_{i}$ and $\hbar_{i \propto c}$ chosen positive constants appropriately, where $i=1,2, \ldots N$.Then $\hbar_{i}(t)$ satisfies:

(1) $\hbar_{i}(t)=\operatorname{csch}\left(\kappa_{i} t+\theta_{i}\right)+\hbar_{i \propto}=\frac{2}{e^{t_{i}}-1}+\hbar_{i \propto}>\hbar_{i \propto}$

(2) $\lim _{\theta_{i} \rightarrow+\infty} \hbar_{i}(0) \rightarrow+\propto ;(3) \lim _{t \rightarrow+\infty} \hbar_{i}(t)=\hbar_{i \propto \infty}$.

Consider tracking error $e_{i}(t)$, prescribed performance is achieved if the following inequality is satisfied.

$$
-\underline{\delta}_{i} \hbar_{i}(t)<e_{i}(t)<\bar{\delta}_{i} \hbar_{i}(t)
$$

Where $\underline{\delta}_{i}, \bar{\delta}_{i}$ are positive constants associated with $e_{i}(t), \quad i=1,2, \ldots, N$.

The performance bound is constrained by $(4), e_{i}(t)$ is shown in Fig.4. When $\theta_{i}$ is chosen adequately small, and $\underline{\delta}_{i}, \bar{\delta}_{i}$ are positive, we can easily get $-\underline{\delta}_{i} \hbar_{i}(0) \rightarrow-\propto$ and $\bar{\delta}_{i} \hbar_{i}(0) \rightarrow+\propto$. So, for a bounded $e_{i}(0)$ and an adequately small $\theta_{i}$, the following inequality is acquired.

$$
-\underline{\delta}_{i} \hbar_{i}(0)<e_{i}(0)<\bar{\delta}_{i} \hbar_{i}(0)
$$

Appropriately, with the aforesaid proposed performance function(3), there is no need to get accurate initial error $e_{i}(0)$.

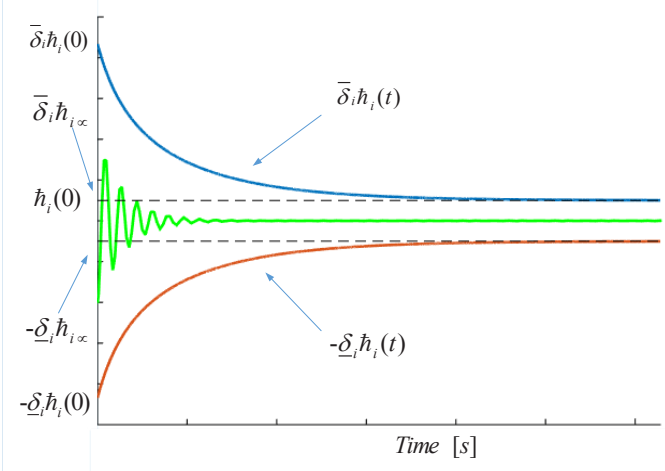

Figure 4. The PPB on the error $e_{i}(t)$

\section{Remark1}

1. Provided that $-\underline{\delta}_{i} \hbar_{i}(0)<e_{i}(0)<\bar{\delta}_{i} \hbar_{i}(0)$, $\underline{\delta}_{i} \hbar_{i \propto}, \bar{\delta}_{i} \hbar_{i \infty}$ determine the maximum allowable size of the error and the steady state. The parameter $\kappa_{i}$ domains the decreasing rate of $\hbar_{i}(t)$, which will domain the decreasing rate bound of $e_{i}(t)$ when the overshoot is no more than $\underline{\delta}_{i} \hbar_{i}(0), \bar{\delta}_{i} \hbar_{i}(0)$. All parameters mentioned above can be arbitrary regulated.

2. The performance function applied in $[17,18]$ is imposed by $\rho(t)$, the tracking error $e(t)$ is given as follows:

$$
\left\{\begin{array}{l}
-\delta_{1} \hbar_{i}(t)<e(t)<1, \quad \text { if } e(0)>0 \\
-1<e(t)<\delta_{2} \hbar_{i}(t), \quad \text { if } e(0)<0
\end{array}\right.
$$

with $0 \leq \delta_{1} \leq 1,0 \leq \delta_{2} \leq 1$. Appropriately, the behavior bounds are different with different initial value of the error. We can also see that the controller design and stabilized analysis are supposed to differ because of different initial value. Additionally, acquirement of the $e(0)$ adds the difficulty of algorithm applying.

Considering that it is unable to devise controller directly based on q.(4), an error transfer function $\mu\left(\varepsilon_{i}\right)$ is introduced to transfer the "constrained" system into equivalent "unconstrained" system.

$$
e_{i}(t)=\mu_{i}\left(\varepsilon_{i}\right) \hbar_{i}(t)
$$

With

$$
\mu\left(\varepsilon_{i}\right)=\frac{\bar{\delta}_{i} e^{\varepsilon_{i}}-\underline{\delta_{i} e^{-\varepsilon_{i}}}}{e^{\varepsilon_{i}}+e^{-\varepsilon_{i}}}
$$

Where $\varepsilon_{i}, i=1,2, \ldots, N$ donates transfer error. $\mu\left(\varepsilon_{i}\right)$ satisfies:

(1) $\mu\left(\varepsilon_{i}\right)$ is smooth and strictly increasing;

(2) $\lim _{\varepsilon_{i} \rightarrow-\infty} \mu_{i}\left(\varepsilon_{i}\right)=-\underline{\delta_{i}}, \lim _{\varepsilon_{i} \rightarrow+\infty} \mu_{i}\left(\varepsilon_{i}\right)=\overline{\delta_{i}}$.

Based on the properties of $\mu\left(\varepsilon_{i}\right)$,original error is transferred into

$$
-\underline{\delta_{i}}<\mu_{i}(t)<\overline{\delta_{i}}
$$

If we multiply the above formulas, considering $\hbar_{i}(t)$ is positive, we can get

$$
-\underline{\delta}_{i} \hbar_{i}(t)<e_{i}(t)<\bar{\delta}_{i} \hbar_{i}(t)
$$

Appropriately, (4)is equivalent illustrated by Eq. (7).

Furthermore, since $\mu_{i}\left(\varepsilon_{i}\right)$ is strictly increasing as well as $\hbar_{i}(t) \geq \hbar_{i \propto}>0$, we can get the inverse transformation (7).

$$
\varepsilon_{i}(t)=\mu_{i}^{-1}\left(\varepsilon_{i}\right)=\frac{1}{2} \ln \left(\frac{e_{i} / \hbar_{i}+\delta_{i}}{\overline{\delta_{i}}-e_{i} / \hbar_{i}}\right)
$$

And

$$
\dot{\varepsilon}_{i}(t)=\mathrm{r}_{i}\left(\dot{e}_{i}-\frac{\dot{\hbar}_{i}}{\hbar_{i}} e_{i}\right)
$$

With

$$
\begin{aligned}
& r_{i}=\frac{1}{2}\left(\frac{1}{e_{i} / \hbar_{i}+\underline{\delta_{i}}}-\frac{1}{e_{i} / \hbar_{i}-\overline{\delta_{i}}}\right)>0 \\
& \dot{\hbar}_{i}(t)=\kappa_{i}-\kappa_{i}\left[\operatorname{coth}\left(\kappa_{i} t+\theta_{i}\right)\right]^{2}
\end{aligned}
$$

3.2 Design of prescribed performance controller 
Assumption 1 . The desired trajectory $x_{d}(t)$ is limited and unknown, and $\dot{x}_{d}(t)$ is limited.

The following control scheme is defined

$$
\begin{aligned}
& \mu_{2}(t)=\frac{\tilde{x}_{1}}{\hbar_{2}(t)} \\
& a_{2}=-k_{1} \varepsilon_{1} \\
& \mu_{1}(t)=\frac{\tilde{x}_{2}}{\hbar_{1}(t)} \\
& a_{2}=-k_{2} \varepsilon_{2} \\
& u=a_{3}
\end{aligned}
$$

with

$$
\left\{\begin{array}{l}
\tilde{x}_{1}=\tilde{\dot{\theta}}_{l}=\dot{\theta}_{l}-\dot{\theta}_{d}=x_{1}-x_{d} \\
\tilde{x}_{2}=\tilde{T}_{c}=T_{c}-T_{d}=x_{2}-a_{2} \\
\varepsilon_{1}=\ln \left(\frac{\mu_{1}(t)+\underline{\delta_{1}}}{\overline{\delta_{1}}-\mu_{1}(t)}\right) \\
\varepsilon_{2}=\ln \left(\frac{\mu_{2}(t)+\underline{\delta_{2}}}{\overline{\delta_{2}}-\mu_{2}(t)}\right) \\
\hbar_{1}(t)=\operatorname{csch}\left(k_{3} t+\theta_{1}\right)+\hbar_{1 \propto} \\
\hbar_{2}(t)=\operatorname{csch}\left(k_{4} t+\theta_{2}\right)+\hbar_{\propto}
\end{array}\right.
$$

Where $k_{1}>0, k_{2}>0$ are design constants of proportion controlling coefficient, $u$ is the controller input.

Through inspection of Eqs. (14)-(15), it is easy to find that the control law doesn't need any derivatives of $x_{i}$ and $a_{i}$, so the controller design and implementation are greatly simplified.

\subsection{Stabilized analysis}

Assumption 2. There is a bounded constant $B_{f}$, satisfying $\frac{\partial F_{f}\left(x_{1}\right)}{\partial x_{1}} \geq B_{f}$

Assumption 3 . The velocity disturbance $\Delta$ is limited.

Theorem 1. Consider the closed-loop system consisting of stabilized platform with controllerEq.(14), all the signals involved are uniformly ultimately bounded.

Choose the following Lyapunov function as

$$
V=V_{1}+V_{2}
$$

with

$$
V_{1}=\frac{1}{2} \varepsilon_{1}^{2}, \quad V_{2}=\frac{1}{2} \varepsilon_{2}^{2}
$$

Substituting time derivative of $V$ into Eq. (11), we can get

$$
\begin{aligned}
\dot{V} & =\dot{V}_{1}+\dot{V}_{2} \\
& =\varepsilon_{1} \dot{\varepsilon}_{1}+\varepsilon_{2} \dot{\varepsilon}_{2} \\
& =\varepsilon_{1} \cdot \frac{2 \dot{\mu}_{1}(t)}{1-\mu_{1}^{2}(t)}+\varepsilon_{2} \cdot \frac{2 \dot{\mu}_{2}(t)}{1-\mu_{2}^{2}(t)}
\end{aligned}
$$

where

$$
\dot{\mu}_{1}(t)=\frac{\dot{\tilde{x}}_{1} \cdot \hbar_{1}(t)-\tilde{x}_{1} \cdot \dot{\hbar}_{1}(t)}{\hbar_{1}^{2}(t)}
$$

Combining with Eq.(1), we can get

$$
\dot{\mu}_{1}(t)=\frac{x_{2} / J_{L}-F_{f}\left(x_{1}+\Delta\right)-\dot{x}_{1 d}-\dot{\hbar}_{1}(t) \cdot \mu_{1}(t)}{\hbar_{1}(t)}
$$

Furthermore, with (18) we have

$$
\begin{aligned}
\dot{V}_{1}= & \frac{2 \varepsilon_{1}(t)\left(\left(-k_{1} \varepsilon_{1}+\hbar_{2}(t) \cdot \mu_{2}(t)\right) / J_{L}\right)}{\hbar_{1}(t)\left(1-\mu_{1}^{2}(t)\right)} \\
& \frac{-F_{f}\left(x_{1}+\Delta\right)-\dot{x}_{1 d}-\dot{\hbar}_{1}(t) \cdot \mu_{1}(t)}{\hbar_{1}(t)\left(1-\mu_{1}^{2}(t)\right)}
\end{aligned}
$$

According to Assumption 3, and within the metabolic range of $x_{1}, F_{f}\left(x_{1}+\Delta\right)$ is limited. $\dot{x}_{1 d}, \dot{\hbar}_{1}(t), \hbar_{2}(t), \mu_{2}(t)$ are limited too.

Define a limited constant $F_{1}$, it is obvious that $\left|\hbar_{2}(t) \cdot \mu_{2}(t) / J_{L}-F_{f}\left(x_{1}+\Delta\right)-\dot{x}_{1 d}-\dot{\hbar}_{1}(t) \cdot \mu_{1}(t)\right| \leq F_{1}(20)$

Moreover,

$$
\begin{aligned}
\dot{V}_{1} & \leq \frac{2\left|\varepsilon_{1}(t)\right| \cdot \bar{F}_{1}}{\hbar_{1}(t)\left(1-\mu_{1}^{2}(t)\right)}-\frac{2 \varepsilon_{1}^{2}(t) \cdot k_{1}}{\hbar_{1}(t)\left(1-\mu_{1}^{2}(t)\right)} \\
& =\frac{2\left|\varepsilon_{1}(t)\right|\left(\bar{F}_{1}-k_{1}\left|\varepsilon_{1}(t)\right| / J_{l}\right)}{\hbar_{1}(t)\left(1-\mu_{1}^{2}(t)\right)}
\end{aligned}
$$

Define

$$
\Omega_{\varepsilon_{1}}=\left\{\varepsilon_{1}|| \varepsilon_{1}(t) \mid \leq \frac{J_{l} \bar{F}_{1}}{k_{1}}\right\}
$$

It is obvious that $\dot{V}_{1}$ will be negative if $\varepsilon_{1} \notin \Omega_{\varepsilon_{1}}$. Thus, $e_{1}$ is bounded.

Similarly, combined with Eq. (1) and Eq. (14), the following equation is acquired.

$$
\begin{aligned}
\dot{\mu}_{2}(t)= & \frac{-\frac{C_{m} C_{e}}{L_{a}} x_{1}-\frac{R_{a}}{L_{a}} x_{2}+\frac{C_{m} k_{P W M}}{L_{a}} u}{\hbar_{1}(t)} \\
& \frac{-\frac{C_{e} C_{m}}{L_{a}} \Delta-\dot{x}_{2 d}-\dot{\hbar}_{2}(t) \cdot \mu_{2}(t)}{\hbar_{1}(t)}
\end{aligned}
$$

Combining with Eq. (14) and Eq. (23), We can get

$$
\begin{aligned}
\dot{V}_{2}= & \frac{2 \varepsilon_{2}(t)\left(-\frac{C_{e} C_{m}}{L_{a}} x_{1}-\frac{R_{a}}{L_{a}} x_{2}-\frac{C_{e} C_{m}}{L_{a}} \Delta\right.}{\hbar_{2}(t)\left(1-\mu_{2}^{2}(t)\right)} \\
& \frac{\left.-k_{1} \dot{\varepsilon}_{1}-\frac{C_{m} k_{P W M}}{L_{a}} k_{2} \varepsilon_{2}-\dot{\hbar}_{2}(t) \cdot \mu_{2}(t)\right)}{\hbar_{2}(t)\left(1-\mu_{2}^{2}(t)\right)}
\end{aligned}
$$


Table 1. Model Parameter.

\begin{tabular}{ccccccccccc}
\hline Symbol & $J_{L}$ & $C_{m}$ & $L_{a}$ & $R_{a}$ & $k_{P W M}$ & $C_{e}$ & $F_{C}$ & $F_{S}$ & $B_{V}$ & $v_{s}$ \\
\hline Parameter & 0.0012 & 0.625 & 0.062 & 5.1 & 3.75 & 0.75 & 0.2 & 0.3 & 0.017 & 0.2 \\
\hline
\end{tabular}

Choosing step signal as the reference signal, Fig.5

According to Eq.(21), $x_{1}$ is limited. Based on the Singular value theory, $x_{2}$ is limited. Considering that $\Delta, \dot{\hbar}_{2}(t)$ are limited, invoking Eq. (12), $\dot{\varepsilon}_{1}$ is limited.

Define a limited constant $F_{2}$, it is obvious that

$$
\left|-\frac{C_{e} C_{m}}{L_{a}} x_{1}-\frac{R_{a}}{L_{a}} x_{2}-\frac{C_{e} C_{m}}{L_{a}}-k_{1} \dot{\varepsilon}_{1}-\Delta \dot{\hbar}_{2}(t) \cdot \mu_{2}(t)\right| \leq F_{2}
$$

Moreover,

$$
\begin{aligned}
\dot{V}_{1} \leq & \frac{2\left|\varepsilon_{2}(t)\right| \cdot \bar{F}_{2}}{\hbar_{2}(t)\left(1-\mu_{2}^{2}(t)\right)}-\frac{2 C_{m} k_{P W M} \cdot k_{2} \varepsilon_{2}{ }^{2}(t)}{L_{a} \hbar_{2}(t)\left(1-\mu_{2}^{2}(t)\right)} \\
= & \frac{2\left|\varepsilon_{2}(t)\right|\left(\bar{F}_{2}-\left|\varepsilon_{2}(t)\right| C_{m} k_{P W M} \cdot k_{2} / L_{a}\right)}{\hbar_{2}(t)\left(1-\mu_{2}^{2}(t)\right)} \\
& \text { Define } \Omega_{\varepsilon_{2}}=\left\{\varepsilon_{2}|| \varepsilon_{2}(t) \mid \leq \frac{L_{a} \bar{F}_{2}}{C_{m} k_{P W M} \cdot k_{2}}\right\}
\end{aligned}
$$

It is obvious that $\dot{V}_{2}$ will be negative if $\varepsilon_{2} \notin \Omega_{\varepsilon_{2}}$.

Thus, $e_{2}$ is bounded.

Thus, all the signals involved are uniformly ultimately bounded. This is the end of proof.

Remark2. By Theorem 1, the bound of $\varepsilon_{1}$ and $\varepsilon_{2}$ are achieved. So we can conclude that the velocity tracking error and the moment tracking error can converge to the prescribed performance bound.

\section{Simulation results}

In this section, simulations are carried out to verify the effectiveness of the proposed control scheme for stabilized platform. The plant is stabilized platform of seeker.

The simulation step size is chosen as $T=0.001 \mathrm{~s}$.

The parameters of the control system are given in Table 1.

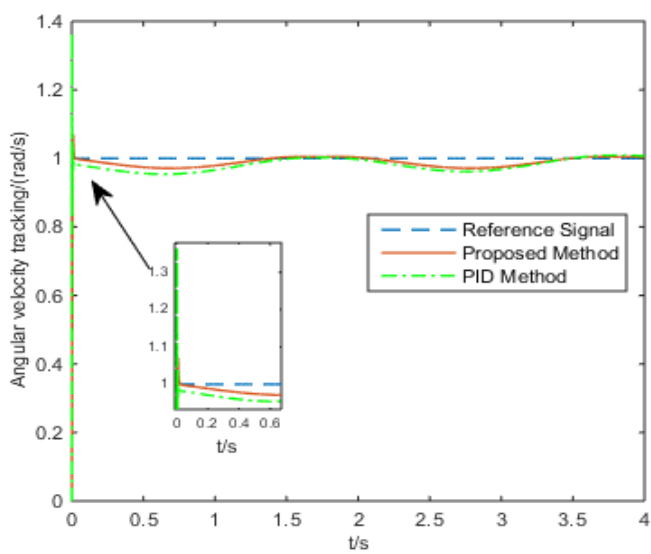

Figure 5. Angular velocity tracking response shows the response of angular velocity tracking with the velocity disturbance chosen as $\sin (3 t-0.5)(\mathrm{rad} / \mathrm{s})$. From Fig.5, we can see that the proposed method responds quickly, and it has smaller overshoot and smaller tracking error than PID method.

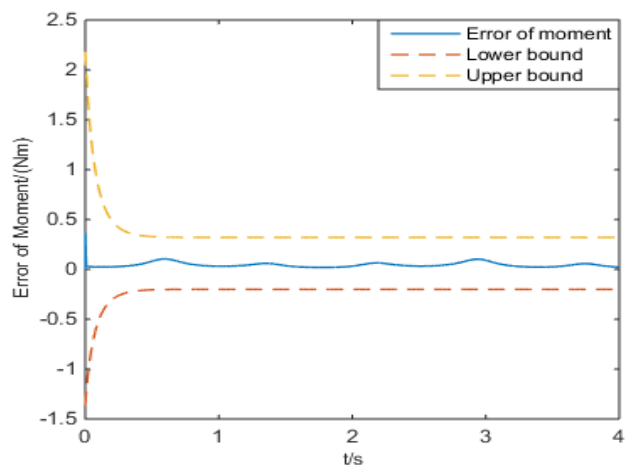

(a) Output error of moment with parameter perturbation

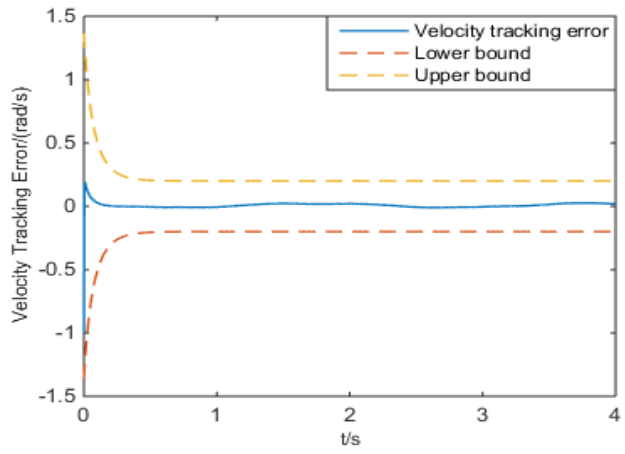

(b) Output error velocity with parameter perturbation

Figure 6. Response of system state variety with step signal

To verify the robustness of the proposed method, $50 \%$, perturbation is applied to coefficient $\frac{1}{J_{L}},-\frac{C_{m} C_{e}}{L_{a}},-\frac{R_{a}}{L_{a}}$.

From Fig.6, we can see that the error of states converges to zero with acceptable accuracy, Under the condition of intense parameter perturbation and angular velocity disturbance, the steady deviation of the response is no more than $3 \%$.

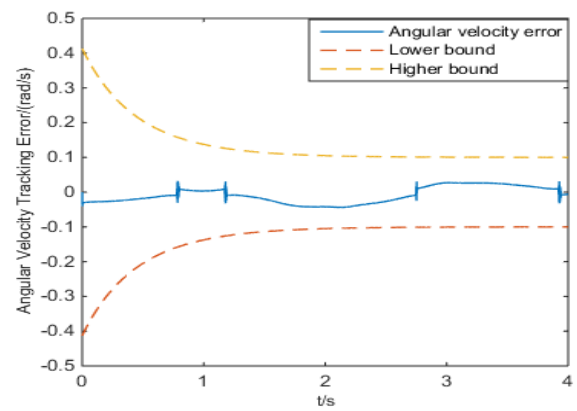

Figure 7. Response of system state variety of sine signal 
To verify the control effect of the plant's angular velocity crossing the zero point frequently, sine signal is chosen as the reference signal, with the disturbance chosen as $\cos (3 t)(\mathrm{rad} / \mathrm{s})$, similarly, $50 \%$ parameter perturbation is introduced.

We can see from Fig.7 that the stabilized platform is capable of achieving prescribed bounds on the transient and steady state output error performance, through observation of Fig.7, it is easy to find that the system achieves more than $95 \%$ isolation of internal and external disturbance.

Remark3. The proposed controller doesn't reside to the construction of friction model or special analytical friction expressions, it has promoting value in engineering practice with its easy design structure and relaxed application condition.

\section{Conclusion}

In this paper, a prescribed performance controller, which allows transient performance and steady value of moment and angular velocity tracking errors to achieve prescribed bound, is addressed for stabilized platform with Stribeck friction. The designed performance function takes satisfying effect without acquiring the initial error. It's verified that the plant using the proposed method responses rapidly. And it achieves high-precision tracking with parameter perturbation and unknown disturbance. Besides, the phenomenon of speed dead-area is eliminated by the proposed method. Finally, because of its simple design structure and its loose applying condition, it has promoting value in engineering practice.

\section{References}

1. Haarhoff D, Kolditz M, Abel D, et al. Actuator design for stabilizing single tendon platforms. 23rd International Conference on Robotics, 46,371378.(2017)

2. Stepaniak, MJ, de Haag, MU,Van Graas, F, Field programmable gate array-based attitude stabilization. Journal of aerospace computing information and communication. 6(7),451-463. (2009)

3. Song XR, Chen H,Xue YG, Stabilization precision control methods of photoelectric aim-stabilized system. 351,115-120. (2015)

4. Liu F, Wang H, Fuzzy PID controller for two-axis airborne optoelectronic stabilized platform. International journal of computing information and control, 13(4), 1307-1322.(2017)

5. Kennedy P J, Kennedy R L, Direct versus indirect line of sight stabilization. IEEE Transactions on Control System Technology, 11(8), 1293-1299. (2003)

6. Sun, H., Zhang, S. M.,Computation model and error budget for image motion of aerial imaging system. Electronics Optics\& Control, 20(11), 2492-2499. (2012)

7. Zhang YS., Yang T., Li CY., Liu SS., Du CC., Liu M., et al. Fuzzy-PID control for the position loop of aerial inertially stabilized platform. Aerospace Sci Technology, 36, 21-26.(2014)

8. Martin R, Zdenek H, Structured MIMO design for dual-stage inertial stabilization: case study for HIFOO and Hinfstruct solvers. Mechatronics ,23(8),1084-1093.(2013)

9. Zou Y., Lei XS., A compound control method based on the adaptive neural network and sliding mode control for inertial stable platform. Neurocomputing,155,286-294.(2015)

10. ZhouXY., Yuan JQ., Cai TT., Dual-rate-loop control based on disturbance observer of angular acceleration for a three-axis aerial inertially stabilized platform. ISA Transactions, 63, 288-298. (2016)

11. Ekstrand B, Equations of motion for a two-axes gimbal system. IEEE TransAerospace Electron System, 37(3),1083-1091.(2001)

12. Bechlioulis C, Rovithakis G, Robust adaptive control of feedback linearizable MIMO nonlinear systems prescribed performance. IEEE Trans. Automation Control,53(9),2090-2099.(2008)

13. Zhao K, Song Y D, Ma T D, et al. Prescribed performance of uncertain Euler-Lagrange system subject to full-state constraints. IEEE Transactions on Neural Networks and Learning System, To be punished, doi: 10.1109/TNNLS.2017.2727223.

14. Zhang Z Y, Li Z Q, Zhou Q K, Application in prestiction friction compensation for angular velocity loop of inertially stabilized platforms. Chinese Journal of Aeronautics, 27(3),655-662. (2014)

15. Wang Y S, Wang Q J, Shi F H, Development of a set of Stribeck curves for conformal contacts of rough surfaces. Tribology\& Lubrication Technology, 63(4),32-40.(2007)

16. Bechlioulis, Charalampos P, Rovithakis, George A, Prescribed performance adaptive control for multiinput multi-output affine in the control nonlinear systems. IEEE Transactions on automatic control, 55(5),1220-1226.(2010)

17. Li, D., Liu G P., Hua C C., Modeling and prescribed performance control for cold rolling mill HAGC system with measurement delay, Proceedings of the 36th Chinese Control Conference, 726-731. (2017)

18. A. Theodorakopoulos and G. Rovithakis, Prescribed performance control of strict-feedback system with hysteresis input nonlinearity. 2015 European Control Conference,3220-3225.(2015) 